\title{
Cisplatin-Gemcitabine Related Cardiomyopathy in Non-Small Cell Lung Cancer NSCLC Patient: A Case Study
}

\section{Dawoud RA ${ }^{*}$, Hind Elmalik ${ }^{2}$ and AL-Azzazy $S^{2}$}

${ }^{1}$ Department of Pharmacy, National Center of Cancer Care and Research, Doha, Qatar

${ }^{2}$ Department of Medical Oncology, National Center of Cancer Care and Research, Doha, Qatar

*Corresponding author: Dawoud RA, Department of Pharmacy, National Center of Cancer Care and Research, Doha, Qatar, Tel: 0097466934413, E-mail: rdawoud@hamad.qa

Citation: Dawoud RA, Hind Elmalik, AL-Azzazy S (2018) Cisplatin-Gemcitabine Related Cardiomyopathy in Non-Small Cell Lung Cancer NSCLC Patient: A Case Study. J Case Rep Stud 6(4): 405.

doi: 10.15744/2348-9820.6.405

Received Date: June 11, 2018 Accepted Date: August 21, 2018 Published Date: August 23, 2018

\begin{abstract}
Gemcitabine is a pyrimidine analog and cisplatin is a platinum agent, they are usually combined to form a chemotherapeutic doublet used to treat different types of oncological cancers, including non-small cell lung cancer (NSCLC). Although these agents are not known to cause such events, few cases related to possible cardiotoxicity side effects have been reported ; including angina, chronic heart failure, arrhythmias, and cardiac ischemia. A 52-year-old African male presented with Stage IV lung adenocarcinoma metastatic to the bone, pleura and lymph node, EGFR wild type. Patient has a history of hypertension, diabetes, and atrial fibrillation and on rate control medications. He was treated initially with cisplatin-gemcitabine, after which he developed cardiomyopathy. This was proven by a two-dimensional echocardiography with an ejection fraction dropping from $65 \%$ to $35 \%$. In our perspective, cardiomyopathy could be related to cisplatin-gemcitabine, which is a rare but potential risk factor for developing cardiomyopathy especially if the patient has pre-existing comorbidities that may potentiate toxicity. An instant discontinuation with close monitoring is needed. Furthermore, we suggest considering other options of chemotherapy for patients if they develop cardiac adverse events.
\end{abstract}

Keywords: Cisplatin-Gemcitabine; Adverse Drug Reactions; Anthracyclines; Cardiomyopathy; Pharmaco-Epidemiology; Platinum Chemotherapy; Cardiotoxicity

\section{Introduction}

Cardiomyopathy is defined as a disease of heart muscles with different etiologies [1]. Patients with solid or hematological malignancies who are treated with chemotherapeutic agents are at increased risk of having cardiovascular side effects, especially if they have added predisposing factors or a known history of heart conditions such as hypertension ischemic heart disease or valvular disease [2].

Non-Small Cell Lung Cancer (NSCLC) is one of the leading causes of death worldwide, which is commonly diagnosed in the advanced stages. In inoperable metastatic cases, platinum-based combination chemotherapies are the treatment of choice in such groups [3]. Many multicenter randomized clinical trials have been conducted to study the benefit of different chemotherapeutic agents like gemcitabine, docetaxel, vinorelbine, paclitaxel, or pemetrexed in the treatment of NSCLC either alone or in combination with cisplatin or carboplatin-based chemotherapy regimens. The outcomes of these trials have been published over the past few years and have demonstrated non-superiority in efficacy of any combination to the others [4].

The combination of cisplatin-gemcitabine is one of those combinations to treat NSCLC. Although these agents are not known to cause cardiac toxicity, few cases have been reported, related to possible cardiotoxicity side effects, including angina, chronic heart failure, arrhythmias, or cardiac ischemia. The mechanism by which these agents might cause such toxicity may be due to electrolyte abnormality [5]. Moreover, the yield of these toxicities could be lethal and worth recognizing.

This case was introduced and treated in the National Center for Cancer Care and Research which is the main cancer center in Qatar, and one of 12 hospitals of Hamad Medical Corporation.

\section{Case Presentation}

Our case is a patient that was initially in good health and performance status. He was active and mobile until May 2017 when he experienced an episode of chest pain associated with a syncopal attack. He was evaluated and cardiac causes were ruled out. After a recurrent episode of blurred vision associated with loss of consciousness, excessive sweating, and dizziness with no reported jerky 
movement, he was evaluated by his primary oncologist abroad and found to have cervical lymphadenopathy. An initial lymph node biopsy confirmed the diagnosis of metastatic squamous cell bronchogenic carcinoma and PET/computed tomography was done abroad, showing metabolically active right upper lung lobe central neoplasm (which was likely Bronchogenic Carcinoma) with lymph nodes, osseous and plural deposits.

On June 2017, the patient was admitted to Hamad General Hospital in the state of Qatar, complaining of right-sided chest pain with syncopal attacks two weeks earlier. A chest x-ray was done and showed right upper lung opacity. The oncology team was consulted and recommended a CT angiography to be done; if normal CT the patient can be discharged to follow-up in 48 hours in the Oncology Clinic.

Upon further questioning, the patient complained of left-sided chest pain radiating to his whole spine and shoulders which increases by pressure and movement. Additionally, he also presented with whitish sputum production accompanied with on/ off cough but no hemoptysis. He had mild shortness of breath and reported a recent history of dysphagia to solid food over the last two months, resulting in anorexia and weight loss of about $10 \mathrm{~kg}$ over the span of two months. The patient was started on antibiotics for suspected pneumonia.

Pretreatment echocardiogram showed normal global systolic left ventricular function, 65 percentile ejection fractions with moderate pericardial effusion without a need for cardiac intervention. Furthermore, patient devolved atrial fibrillation secondary to his lung condition, and started on metoprolol for rate control.

On the $9^{\text {th }}$ of July 2017 , the patient was started on cisplatin-gemcitabine $\left(75 \mathrm{mg} / \mathrm{m}^{2}\right.$ on day $1-1250 \mathrm{mg} / \mathrm{m}^{2}$ day 1 and 8$)$. On day 3 of chemotherapy, patient experienced rapid atrial fibrillation with a heart rate of $147 \mathrm{bpm}$ confirmed with an electrocardiography results (Figure 1A). A new echocardiography (2D ECHO) was done and showed a reduction in the ejection fraction to 33\% with moderate pericardial effusion. The cardiologist impression was that it is a chemotherapy-related event. Repeated electrocardiography was done later to consider inferior ischemia (Figure 1B), Cisplatin-gemcitabine was discontinued, and day 8 of the first cycle was not given. Metoprolol dose was increased by the cardiologist at that time then changed to bisoprolol for the rate control, and angiotensin-converting enzyme inhibitor and diuretics were started as well.
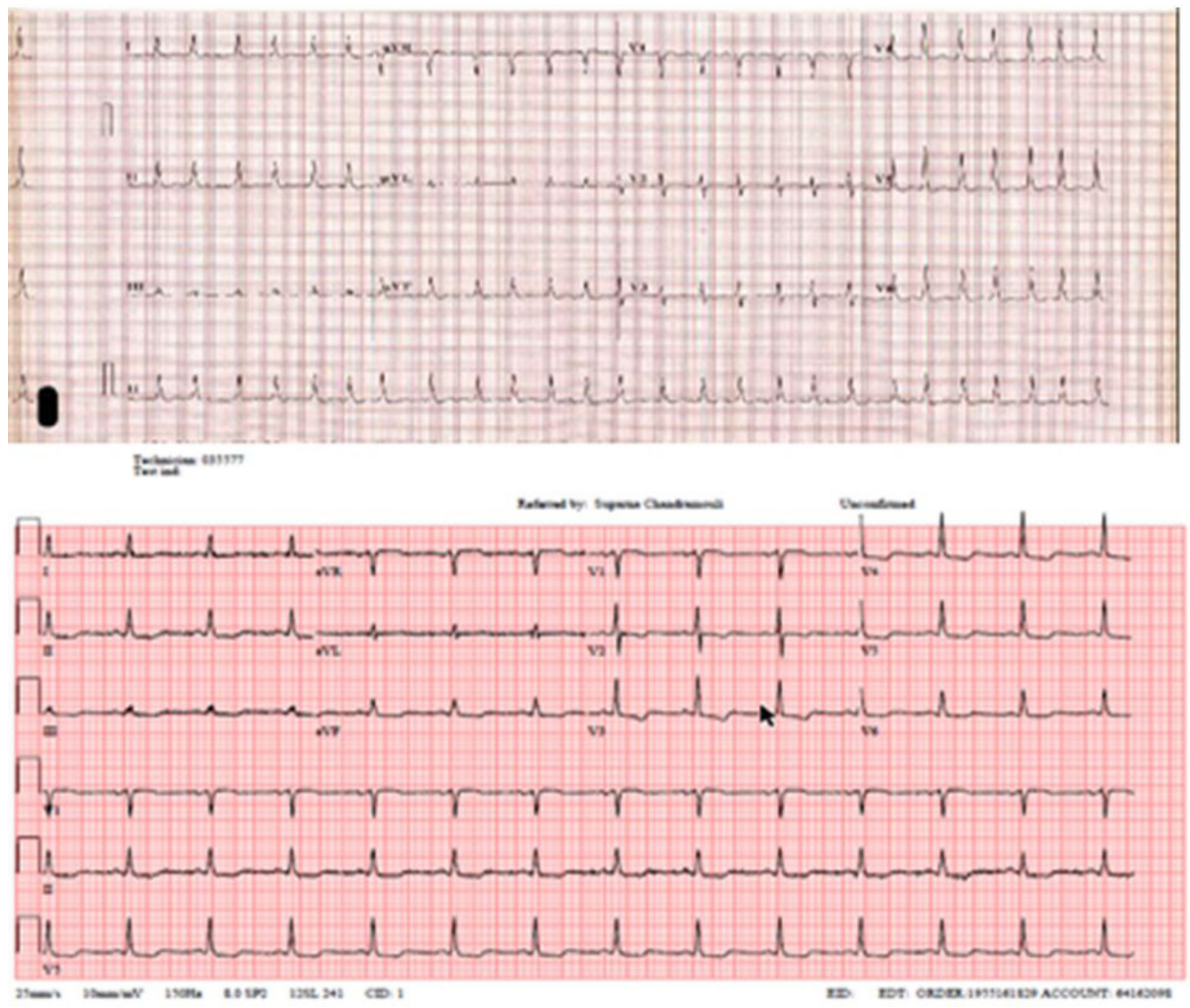

Figure 1: Electrocardiography results (A) Rapid atrial fibrillation; (B) Inferior ischemia

A new cervical lymph node biopsy was done, showing a new diagnosis of metastatic adenocarcinoma of the lung, moderate to poorly differentiated, stage T3N3M1. Plan of care was changed by the thoracic multi-disciplinary team to receive carboplatinpemetrexed regimen and for further cardiac evaluation. The repeated echocardiography revealed an improvement to $40 \%$ left ventricular ejection fraction. His clinical condition was improving and heart rate was controlled; however, his ECOG performance status was III at that time. 
PET/CT was conducted to evaluate the extent of the disease at which showed a large neoplastic mass in the upper lobe of the right lung, causing encasements of the pulmonary artery and vein, in addition to the moderate pericardial effusion confirmed by the echocardiography. Afterwards, the patient became hypotensive and required to start anticoagulation for the atrial fibrillation and pulmonary vein thrombosis that was not initiated due to the drop in hemoglobin and the possibility of doing pericardiocentesis.

The patient was admitted to the cardiac intensive care unit after pericardiocentesis failure and decided to do a pericardial window. The anticoagulation was started and two-dimensional echocardiography ruled out the presence of pericardial effusion and ejection fraction was 65\% (Figure 2). After stabilization and removal of the pericardial drainage, the patient was transferred back to the cancer center for the continuity of care. Furthermore, the immunohistochemistry stains were negative to ALK and EGFR mutation was not detected for the patient using EGFR-RT52 kit.

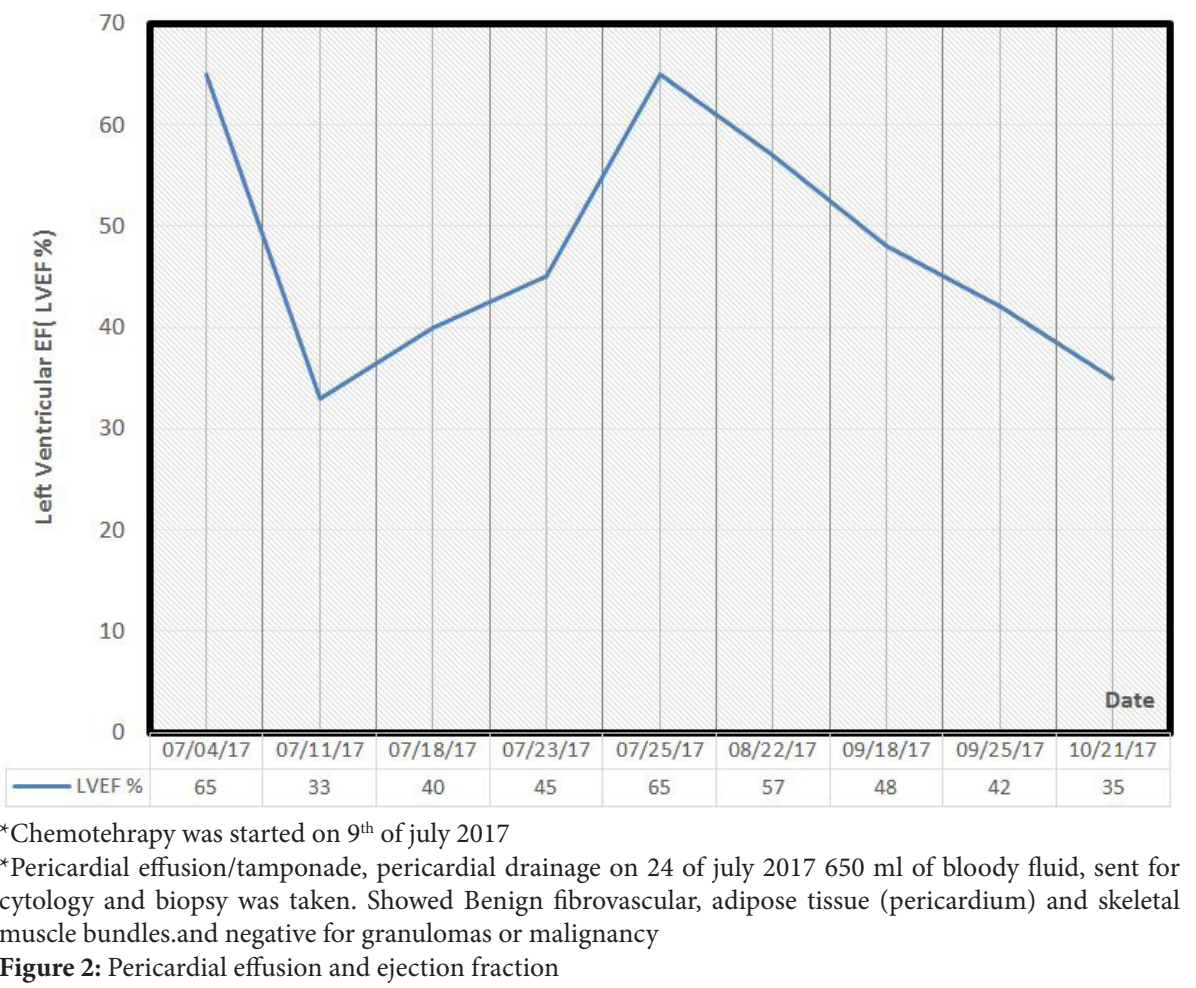

Further chemotherapy was given to the patient, although the patient was informed about the poor general performance status. However, he decided to proceed with the palliative chemotherapy with pemetrexed+carboplatin. He developed disease progression after receiving four cycles of chemotherapy, and his status was changed to Do Attempt Not Resuscitation (DNAR), dying a few days after that.

\section{Discussion}

Cardiotoxicity is a major side effect of several chemotherapeutic agents such as anthracyclines, fluoropyrimidines, cyclophosphamides, and platinum among many others. Cardiovascular manifestations can be considered in hypo-hypertensive changes, electrocardiographic changes, arrhythmias, myocarditis, pericarditis, myocardial infarction, cardiomyopathy, cardiac failure (left ventricular failure) and congestive heart failure [6]. The time frame of the event may be occurring during or shortly after administration of the chemotherapy or years after finishing the treatment.

The Suggested mechanisms by which the cytotoxic agents induce cardiotoxicity are by causing rapid apoptosis or necrosis, growth deprivation and elimination of angiogenesis, or a compromise in repair capacity, for the myocardium and the proliferating cancer cells. Where cisplatin can cause platelets aggregation and thromboxane formation, and more thrombogensis will occur [7].

Gemcitabine-cisplatin is one of the treatment options that are used in NSCLC advanced stage that is not known commonly to cause cardiovascular side effects. However, Wachters et al. reported a reduction in the ejection fraction in one study that compared it to anthracycline-containing regimens up to $2 \%$ [8]. While Yang et al. addressed the toxicities associated with such combination as leukopenia, neutropenia, nausea, vomiting, asthenia, and fatigue but did not mention anything about the cardiotoxic effect of the combination [9]. Furthermore, Tiong et al. reported a case where a patient had no previous cardiovascular comorbidities but had an episode of cardiac failure post carboplatin-gemcitabine in urothelial carcinoma [10]. Bursac et al. studied the incidence of cardiac toxicity in gemcitabine/ cisplatin vs paclitaxel/carboplatin in non-small cell lung cancer patient and concluded that patients with a history of cardiac disease are at higher risk of such events with no statistical significance for the first arm [11]. 
There are many cases reporting cardiomyopathy post cisplatin-gemcitabine or each agent alone. The proposed mechanism of cardiotoxicity was explained for different types of chemotherapeutic agents but was not well mentioned for gemcitabine. However, there are many factors that may contribute to the cardiac event and may be potentiated by the hypercoagulable state introduced by the cancer cells itself, as many of the cytotoxic medications cause thrombosis by their effect on the coagulation pathway or factors which may lead to cardiomyopathy. Moreover, its harmful effects on the structure of the blood vessels increase the risk of thrombosis by impairing the blood flow. Other comorbidities that are common in the elderly population such as atrial fibrillation $(\mathrm{AF})$ and hypertension are treated with medications with antiangiogenic effects [12].

In our case, the patient had predisposing factors to cause cardiotoxicity and hypertension with pre-existing arrhythmia. His ejection fraction, however, was within normal range prior to starting chemotherapy. From there, we can assume the causality and the relationship between the cardiotoxicity and gemcitabine-cisplatin administration. The fluctuation in the ejection fraction preand post-treatment solidifies this assumption of induced cardiomyopathy.

The reduction of the ejection fraction post cisplatin-gemcitabine initiation could be the possible cause of the cardiomyopathy that the patient developed. Additionally, the patient's ejection fraction improved upon discontinuation of the drugs. Although the patient had other risk factors that may contribute to the illness, the causality relation to the chemotherapy-induced cardiomyopathy can be considered in this case.

\section{Conclusion}

Cisplatin-gemcitabine combination might induce cardiomyopathy in patients with pre-existing cardiovascular comorbidities. Further studies are needed to confirm this and identify the mechanism and the possible risks. We suggest considering other chemotherapy alternatives for patients at risk of developing cardiac adverse events.

\section{References}

1. Abelmann WH (1984) Classification and natural history of primary myocardial disease. Prog Cardiovasc Dis 27: 73-94.

2. (1980) Report of the WHO/ISFC task force on the definition and classification of cardiomyopathies. Br Heart J 44: 672-3.

3. Horita N, Nagashima A, Nakashima K, Shibata Y, Ito K, et al. (2017) The best platinum regimens for chemo-naive incurable non-small cell lung cancer: network meta-analysis. Scientific reports 7: 13185

4. Bareschino MA, Schettino C, Rossi A, Maione P, Claudia P, et al. (2011) Treatment of advanced non-small cell lung cancer. J Thorac Dis 3: 122-33.

5. Mortimer JE, Crowley J, Eyre H, Weiden P, Eltringham J, et al. (1992) A phase II randomized study comparing sequential and combined intraarterial cisplatin and radiation therapy in primary brain tumors. A Southwest Oncology Group study. Cancer 695: 1220-3.

6. Yeh ET, Tong AT, Lenihan DJ, Yusuf SW, Swafford J, et al. (2004) Cardiovascular complications of cancer therapy: diagnosis, pathogenesis, and management. Circulation 109: 3122-31.

7. Florescu M, Cintezaa M, Vinereanua D (2013) Chemotherapy-induced Cardiotoxicity. Maedica 8: 59-67.

8. Wachters FM, Van Der Graaf WT, Groen HJ (2004) Cardiotoxicity in advanced non-small cell lung cancer patients treated with platinum and non-platinum based combinations as first-line treatment. Anticancer Res 24: 2079-84.

9. Yang J, He J, Yu M, Li T, Luo L, et al. (2016) The efficacy and safety of platinum plus gemcitabine (PG) chemotherapy with or without molecular targeted agent (MTA) in first-line treatment of non-small cell lung cancer (NSCLC). Medicine (Baltimore) 95: e5599.

10. Tiong FL, Cornillet L, Houede N (2015) Cardiac Failure Caused by the Association of Carboplatin and Gemcitabine Chemotherapy in a Patient with Metastatic Urothelial Cancer: A Case Report. J Clin Case Rep 5: 670.

11. Bursac DS, Sarcev T, Velikic DS, Tepavac A (2016) Cardiotoxic effects of gemcitabine/cisplatin vs paclitaxel/carboplatin first-line chemotherapy in patients with advanced non-small cell lung cancer. Annals of Oncology 27: 411-5.

12. Albini A, Pennesi G, Donatelli F, Cammarota R, De Flora S, et al. (2010) Cardiotoxicity of Anticancer Drugs: The Need for Cardio-Oncology and CardioOncological Prevention. J Natl Cancer Inst 102: 14-25.

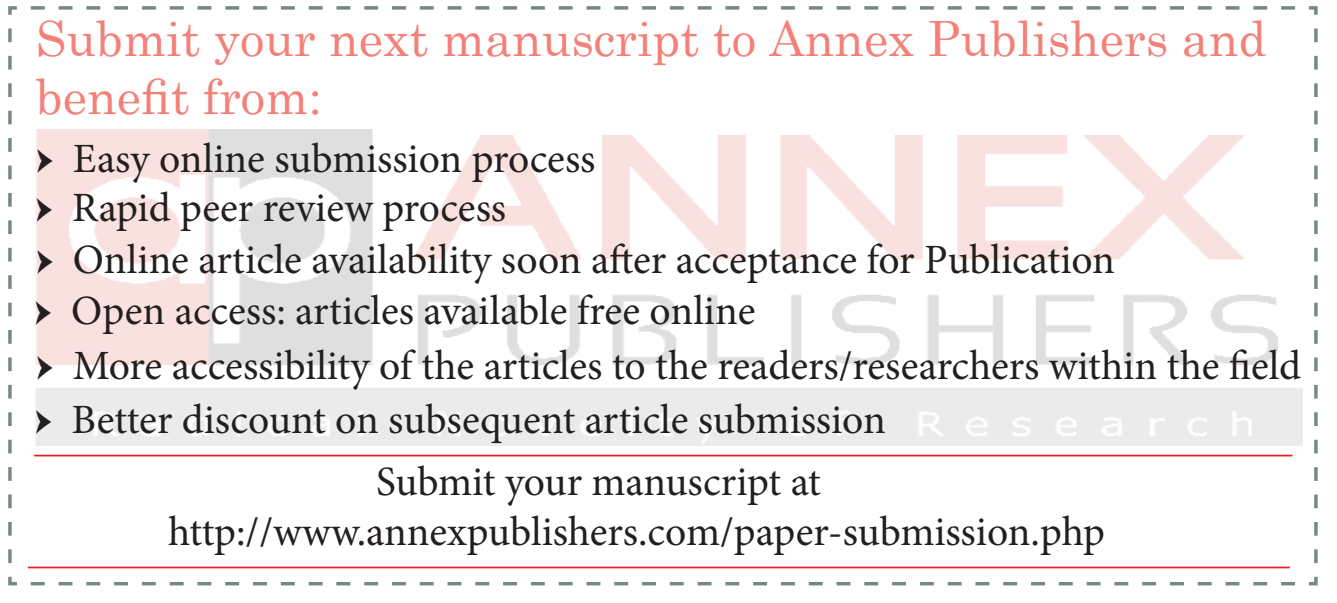

